\title{
Anticancer effect of Ellagic acid and paclitaxel on Lipid Peroxidation and Antioxidant status in female Sprague dawley rats
}

\author{
Sambandham Jamunakumari \\ Department of Medical Biochemistry, Taramani campus, Chennai-600 113
}

\begin{abstract}
There has been growing interest in studying the role played by lipid peroxidation and antioxidant status in breast cancer Free radicals are found to be involved in both initiation and promotion of multistage carcinogenesis. In the present study,effects of both ellagic acid and paclitaxel on the levels of lipid peroxides $(L P O)$ and status of antioxidants in several tissues were studied in mammary carcinoma rats. The levels of reactive oxygen species (ROS) were also measured in control and experimental animals.A significant increase in the levels of LPO,ROS and a decreased levels of antioxidants observed in mammary carcinoma bearing rats were found to be reverted back to near normal levels on treatment with both paclitaxel and ellagic acid.These results suggest that the free radical mediated damage during mammary carcinoma could have been controlled by both ellagic acid and paclitaxel by its free radical quenching and antioxidative potential.The above results also show that both paclitaxel and ellagic acid exert its anticancer effect on the development of breast cancer.
\end{abstract}

Keywords: Ellagic acid, Breast cancer, LPO, Reactive oxygen species.

\section{Introduction}

Breast cancer is the second most prevalent cancer worldwide and their incidence increases gradually. Women who have stage IV breast cancer receive chemotherapy and/or hormonal therapy to destroy cancer cells and control the disease. The goal of chemotherapy is to shrink primary tumors, slow the tumor growth, and to kill cancer cells that may have spread (metastasized) to other parts of the body from the original tumor. Chemotherapeutic drugs elicit some toxicity towards normal cells also, that limits its usage.

Breast cancer incidence is increasing and it is currently estimated that one in eight women develop the disease during lifetime. It is characterized pathologically by a proliferation of malignant epithelial cells within the mammary ductal-lobular system (Dickson and Clarks 1996). Generally, worldwide trends show that in developing countries going through rapid societal and economic changes, the shift towards lifestyles typical of industrialized countries leads to a rising burden of cancers associated with reproductive, dietary, and hormonal risk factors. Incidence has been increasing in most regions of the world, but there are huge inequalities between rich and poor countries. Incidence rates remain highest in more developed regions, but mortality is relatively much higher in less developed countries due to a lack of early detection and access to treatment facilities. For example, in western Europe, breast cancer incidence has reached more than 90 new cases per 100000 women annually, compared with 30 per 100000 in eastern Africa. In contrast, breast cancer mortality rates in these two regions are almost identical, at about 15 per 100 000, which clearly points to a later diagnosis and much poorer survival. Breast cancer is the second leading cause of cancer deaths -about $16 \%$ in women after that of cancer of lungs (Greenlee et al. 2001). About 30\% of the tumors develop in the breast during her 39 years of life and one in every 231 women faces this disease (Landis SH et al.2001). In the United States more than 20,000 women are diagnosed with breast cancer every year, however very few risk prediction tests are known. Approximately $8 \%$ of American women can expect to be stricken with this disease some time during their life time (Moore et al. 1983).

Selective destruction of tumor cells without damaging normal cells is an important goal for cancer chemotherapy. The problem of cancer in the elderly will become increasingly more apparent. Plants, sources of phytochemicals with anti-cancer potential are reported to interfere with targets implicated in carcinogenesis and tumor cell biology makes them interesting tools in cancer research (Morse et al 1993). The carcinogen used in the present study was Dimethyl Benz (a) anthracene. It has been reported to cause cancer in many cancer models like skin, mammary gland, oral, ovarian and salivary gland (Kim et al. 2003). Huggins et al. (1961), described the rat mammary tumor model which is also referred as a'Huggins tumor model' and the histogenesis of the model has been studied by Russo (Russo et al. 1996, 2001). It is an indirect acting carcinogen that requires metabolic activation to yield an ultimate carcinogenic form (Moore et al. 2006).

Flavonoids may interfere in several of the steps that lead to the development of malignant tumors, including protecting DNA from oxidative damage, inhibiting carcinogen activation and activating carcinogen 
detoxifying systems (Ren et al. 2003; Birt et al. 2001). Researchers have become interested in flavonoids for their medicinal properties, especially their potential role in the prevention of cancers.

Ellagic acid is a neutraceutical and has very strong anti-oxidant properties. It is a potent anticarcinogen and anti-mutagenic. It is also considered to be a cancer inhibitor which has the ability to restore apoptosis or normal cell death in cancer cells (Daniel et al. 2001). Paclitaxel is a novel antimicrotubule agent that promotes the assembly of microtubules from tubulin dimers and stabilizes microtubules by preventing depolymerization. This stability results in the inhibition of the normal dynamic reorganization of the microtubule network that is essential for vital interphase and mitotic cellular functions. The purpose of the present study is to evaluate the combined effect of Paclitaxel and Ellagic acid against the DMBA induced mammary carcinogenesis.

Animals:

\section{Materials and methods}

Aged Female Sprague Dawley rats weighing (150-200 g) were purchased from National Institute of Nutrition, Hyderabad, India and were used throughout the study and they were maintained in the controlled environmental conditions of temperature and humidity on alternative $12 \mathrm{hr}$ light/dark cycles. All the animals were fed standard pelleted diet (Gold Mohur rat feed, M/s. Hindustan Lever Ltd., Mumbai) and water ad libitum. Experimental animals were handled according to the university and institutional legislation, regulated by the committee for the purpose of control and supervision of experiments on Animals [CPCSEA, IAEC NO 02/068/06], Ministry of Social Justice and Empowerment, Government of India.

\section{Experimental Protocol :}

The animals were divided into five groups of six animals each.

Group I: Control animals treated with Saline.

Group II: Dimethyl Benz (a) anthracene DMBA treated animals (20 mg $/ \mathrm{kg}$ body wt., one injection by mammary Pad Air pouch technique).

Group III: Breast cancer bearing animals treated with Paclitaxel (33 mg/kg body wt., i.p) for16 weeks (once in a week).

Group IV: Breast cancer bearing animals treated with Ellagic acid (100 mg/kg body wt., orally) for 16 weeks (twice in a week).

Group V: Breast cancer bearing animals treated with both paclitaxel and Ellagic acid (as in Group III and Group IV) for 16 weeks .

\section{Production of Air Pouch}

Air pouch was produced in the Sprague-Dawley rats by the method of Arun et al. (1984). About $2 \mathrm{ml}$ of air was drawn in to the $5 \mathrm{ml}$ syringe. It was autoclaved at $15 \mathrm{psi}$ for 20 minutes in an airtight condition. The sterile air in the syringe was injected carefully just beneath the mammary fat pad subcutaneously so as to produce sterile air pouch. The air inside the pouch was allowed to stabilize for a day before administration of the carcinogen.

\section{Administration of Carcinogen}

$20 \mathrm{mg}$ of DMBA was weighed in a sterile vial and $0.5 \mathrm{ml}$ of sterile saline and $0.5 \mathrm{ml}$ of sunflower oil were added. The vial was stoppered and vortexed vigorously to obtain a uniformly dispersed emulsion. The single dose of DMBA was injected in to the air pouch. The growth of the tumor was measured at frequent intervals up to the $90^{\text {th }}$ day when it attained maximum size. At the end of the experimental period, the animals were killed by cervical decapitation. The breast and liver tissues were used for further analyses.

\section{Biochemical analysis}

Total protein was estimated by the method of Lowry et al . The antioxidant enzymes Superoxide dismutase was analyzed by Marklund and Marklund, Catalase and Glutathione Peroxidase by Sinha and Rotruck respectively. Nonenzymic antioxidants Reduced Glutathione was analyzed by Morom et al, Vitamin E and $\mathrm{C}$ by Desai and Omaye et al respectively. Lipid peroxidation was analysed by Ohkawa .

\section{Statistical analysis}

For statistical analysis, one way analysis of analysis of Variance (ANOVA) was used, followed by the Newman-Keuls Multiple Comparison test. 


\section{Results}

Table I shows the levels of lipid peroxidation, cellular antioxidant enzymes like SOD, CAT and GPx and non-enzymatic antioxidants GSH Vit E and C in Breast tissues of various experimental groups. Highly significant reductions in the activity of enzymic and non-enzymatic antioxidants, with a concomitant increase in the lipid peroxidation in the tumor bearing rats (Group II) were observed. These adverse changes were reversed to near normal levels in Ellagic acid $(100 \mathrm{mg} / \mathrm{Kg}$ body wt.) and Paclitaxel $(33 \mathrm{mg} / \mathrm{Kg}$ body wt.) treated animals (group V) than the animals treated with Paclitaxel (group III) and Ellagic acid alone (group IV) alone.

Fig 1,2,3,4 shows the levels of Lipid peroxidation and enzymic and non- enzymatic antioxidants in liver of various experimental groups. Increase in Lipid peroxidation with significant decrease in antioxidant activities was observed in cancer bearing rats (group II). These changes were reversed in the treatment groups.

\section{Discussion}

Polyunsaturated Fatty acid present in the cell membrane undergoes spontaneous free radical chain reaction in the presence of trace of transition metal ions and produces free radicals (Gutteridge 1990). Lipid peroxidation and free radicals are closely associated with various human diseases such as atherosclerosis, diabetes, neurodegenerative diseases and cancer. Flavonoids for a long time have been part of the herbal treatment that act at the initiation stage of LPO as scavengers which react with peroxyl radicals of PUFA breaking the chain reaction (Ratty 1998).

Significant increase in lipid peroxidation associated with various forms of carcinogenesis has been documented widely and the scavengers are known to play an important role in cancer prevention or treatment. In the present study also significant increase in the levels of lipid peroxidation was observed in the breast and liver tissues of cancer bearing rats. This may be due to the enormous production of reactive oxygen species and free radicals, which are produced in response to exposure to the carcinogen.

One of the prominent and medically most useful properties of flavonoids is their ability to scavenge the free radicals (Cholbi 1991). Paclitaxel eliminates the tumor cells and reduces the tumor burden in group III cancer bearing rats. Also Ellagic acid when given in combination with any drug increases the potential of the accompanying drug(Agarwal 1981). Hence group V animals treated with both Paclitaxel and Ellagic acid show significant decrease in Lipid peroxidation by modulating the glutathione peroxidase- glutathione reductase system. Gallic acid is a structural product of Ellagic acid contributes to the antilipid peroxidative activity. The present study indicates that Ellagic acid with paclitaxel significantly suppress the LPO in breast and liver tissues of cancer bearing rats.

The survival of any organism may depend on its ability to overcome the toxic effect of oxygen species. Antioxidants are the free radical scavengers that provide a natural defense system against free radicals (Sun 1990). The activities of antioxidant enzymes like SOD, CAT and GPx were significantly reduced in the group II cancer bearing rats.

This is due to the increased levels of oxygen radicals which themselves reduce the activity and availability of antioxidants (Wiseman 1996)). Several authors have cited the decreased activities of these antioxidant enzymes in various types of tumors (Winkler 1992)). Group III cancer bearing animals treated with Paclitaxel alone showed increase in the activities of these enzymes when compared to group II animals. This might be due to the cytotoxicity exerted by Paclitaxel towards cancer cells, which inhibits the cell division hence the utilization of these enzymes by the tumor cells. In Pacliatxel and Ellagic acid treated Group V animals the activities were significantly inclined towards near normal due to the combined cytotoxic and antioxidant effect of the drugs used in our present study.

The presence of nonenzymic antioxidants like Reduced glutathione (GSH) Vitamin C and E are also essential for the removal of radicals(Moron 1979) .GPx the major antioxidant enzymes decomposes hydroxyl radicals at the expense of GSH. Hence any decrease in GPx concentration can attribute to the decline in glutathione concentration. In the present study the decreased level of GSH in cancer bearing rats might be due to its irreversible loss following oxidative stress and or use of GSH for free radical scavenging excess utilization of these antioxidant for cell proliferation by tumor cells also . Vitamin $\mathrm{E}$ is a chain breaking antioxidant that donates its labile hydrogen atom from phenolic hydroxyl groups to propagating lipid peroxyl and alkoxyl radical intermediates of LPO, thus terminating the chain reaction(Desai 1984). Reduced glutathione keeps up the cellular levels of ascorbic acid and tocopherol in active form. Hence GSH Vitamin C and E are tightly linked to each other . Reduced glutathione replenish vitamin $\mathrm{E}$ from $\alpha$ tocopherol radical and vitaminC also supports the recycling of tocopherol. When there is reduction in the levels of GSH cellular levels of nonenzymic antioxidants are also lowered. There is extensive evidence that administration of ellagic acid for 16 weeks can increase the GSH level.

In conclusion the antioxidant properties of Ellagic acid could very well be attributed to their free radical scavenging activity against alkoxyl radicals. When given combined with Paclitaxel it reduces the toxic side effects of the later by its immunomodulatory activity and improves the treatment strategy. Our data 
Anticancer effect of Ellagic acid and paclitaxel on Lipid Peroxidation and Antioxidant status in ....

suggests that administration of Ellagic acid $100 \mathrm{mg} / \mathrm{Kg}$ with Paclitaxel significantly decrease the toxic implications of chemotherapy and increases the levels of free radical scavenging enzymes.

Table 1: Effect of Ellagic acid and Paclitaxel on non-enzymic antioxidants induced by DMBA in haemolysate, breast and liver of control and experimental animals

\begin{tabular}{|c|c|c|c|c|c|c|}
\hline & & Group I & Group II & Group III & Group IV & Group V \\
\hline \multirow{3}{*}{$\begin{array}{l}\text { Reduced } \\
\text { Glutathione } \\
\text { (GSH) }\end{array}$} & Haemolysate & $7.93 \pm 0.91$ & $3.47 \pm 0.24 \mathrm{a}^{\#}$ & $5.27 \pm 0.31 \mathrm{~b}^{\#}$ & $5.96 \pm 0.42 b^{\#}$ & $6.55 \pm 0.49 \mathrm{~b}^{\#}$ \\
\hline & Breast & $6.50 \pm 0.53$ & $3.43 \pm 0.27 \mathrm{a}^{\#}$ & $4.50 \pm 0.44 \mathrm{~b}^{\#}$ & $5.63 \pm 0.45 b^{\#}$ & $6.06 \pm 0.53 \mathrm{~b}^{\#}$ \\
\hline & Liver & $4.8 \pm 0.44$ & $2.4 \pm 0.26 \mathrm{a}^{\#}$ & $3.0 \pm 0.31 \mathrm{~b}^{\varrho}$ & $1.82 \pm 0.16 \mathrm{~b}^{@}$ & $4.2 \pm 0.35 \mathrm{~b}^{\#}$ \\
\hline \multirow[b]{3}{*}{ Vitamin C } & Haemolysate & $4.39 \pm 0.91$ & $1.74 \pm 0.77 \mathrm{a}^{\#}$ & $2.66 \pm 0.18 \mathrm{~b}^{\#}$ & $2.96 \pm 0.19 \mathrm{~b}^{\#}$ & $3.39 \pm 0.25 b^{\#}$ \\
\hline & Breast & $3.70 \pm 0.26$ & $1.45 \pm 0.13^{\#}$ & $2.06 \pm 0.10 \mathrm{~b}^{\#}$ & $2.72 \pm 0.22 \mathrm{~b}^{\#}$ & $3.10 \pm 0.17 \mathrm{~b}^{\#}$ \\
\hline & Liver & $2.6 \pm 0.26$ & $1.5 \pm 0.13 \mathrm{a}^{\#}$ & $1.8 \pm 0.16 \mathrm{~b}^{*}$ & $2.12 \pm 0.14 \mathrm{~b}^{\#}$ & $2.40 \pm 0.26 \mathrm{~b}^{\#}$ \\
\hline \multirow[b]{3}{*}{ Vitamin E } & Haemolysate & $3.72 \pm 0.27$ & $1.66 \pm 0.11 \mathrm{a}^{\#}$ & $2.51 \pm 0.18 \mathrm{~b}^{\#}$ & $3.01 \pm 0.26 \mathrm{~b}^{\#}$ & $3.21 \pm 0.25 \mathrm{~b}^{\#}$ \\
\hline & Breast & $10.1 \pm 0.90$ & $6.50 \pm 0.44 \mathrm{a}^{\#}$ & $7.40 \pm 0.62 b^{*}$ & $8.26 \pm 0.67 b^{\#}$ & $9.20 \pm 0.80 \mathrm{~b}^{\#}$ \\
\hline & Liver & $7.3 \pm 0.62$ & $4.26 \pm 0.40 \mathrm{a}^{\#}$ & $5.0 \pm 0.45 \mathrm{~b}^{*}$ & $5.5 \pm 0.44 b^{\#}$ & $6.35 \pm 0.56 \mathrm{~b}^{\#}$ \\
\hline
\end{tabular}

Each value is expressed as mean \pm S.D for six rats in each group.

Units: Haemolysate: GSH, Vitamin C and E: $\mu \mathrm{g} / \mathrm{dl}$.

Breast and liver tissues: GSH, Vitamin C and E: $\mu \mathrm{g} / \mathrm{mg}$ protein.

a: Group II compared with Group I;

b: Group III, Group IV and Group V compared with Group II.

Statistical significance ${ }^{\#} \mathrm{P}<0.001,{ }^{\circledR} \mathrm{P}<0.01,{ }^{*} \mathrm{P}<0.05$.

Figure.1

Effect of Ellagic acid and Paclitaxel on Lipid peroxidation in control

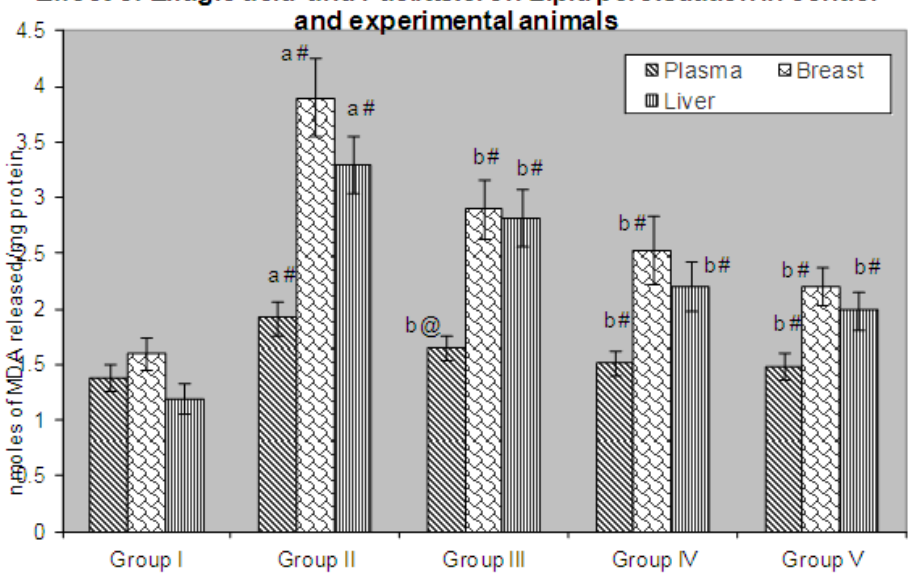

Each value expressed as mean \pm S.D for six animals in each group a: Group II compared with Group I

b:Group III, Group IV and Group V compared with group II

Statistical significance: \#p<0.001,@p<0.01.

Figure.2

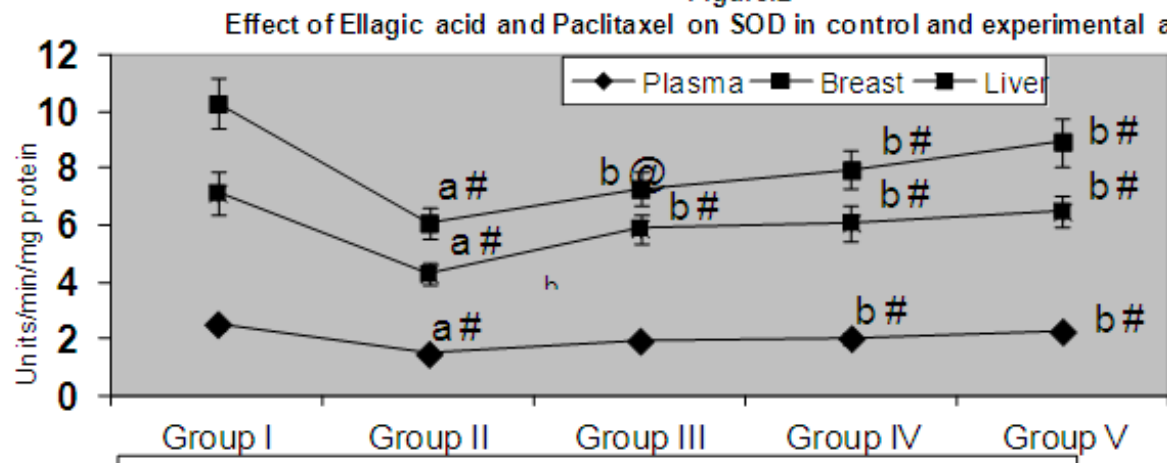

Each value expressed as mean $\pm S$.D for six animal s in each group

a: Group II compared with Group I

b:Group III, Group IV and Group Vcompared with g roup II

Statistical significance: \#p<0.001,@p<0.01. 
Figure.3

Effect of Ellagic acid and Paclitaxel on Catalase in control and

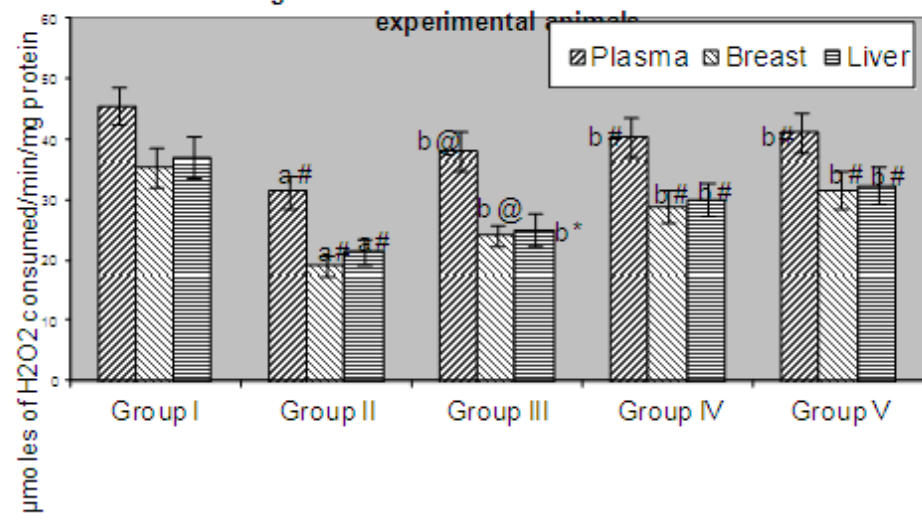

Each value expressed as mean $\pm S . D$ for six animals in each group a:Group II compared with Group

b:Group III, Group IV and Group V compared with group I

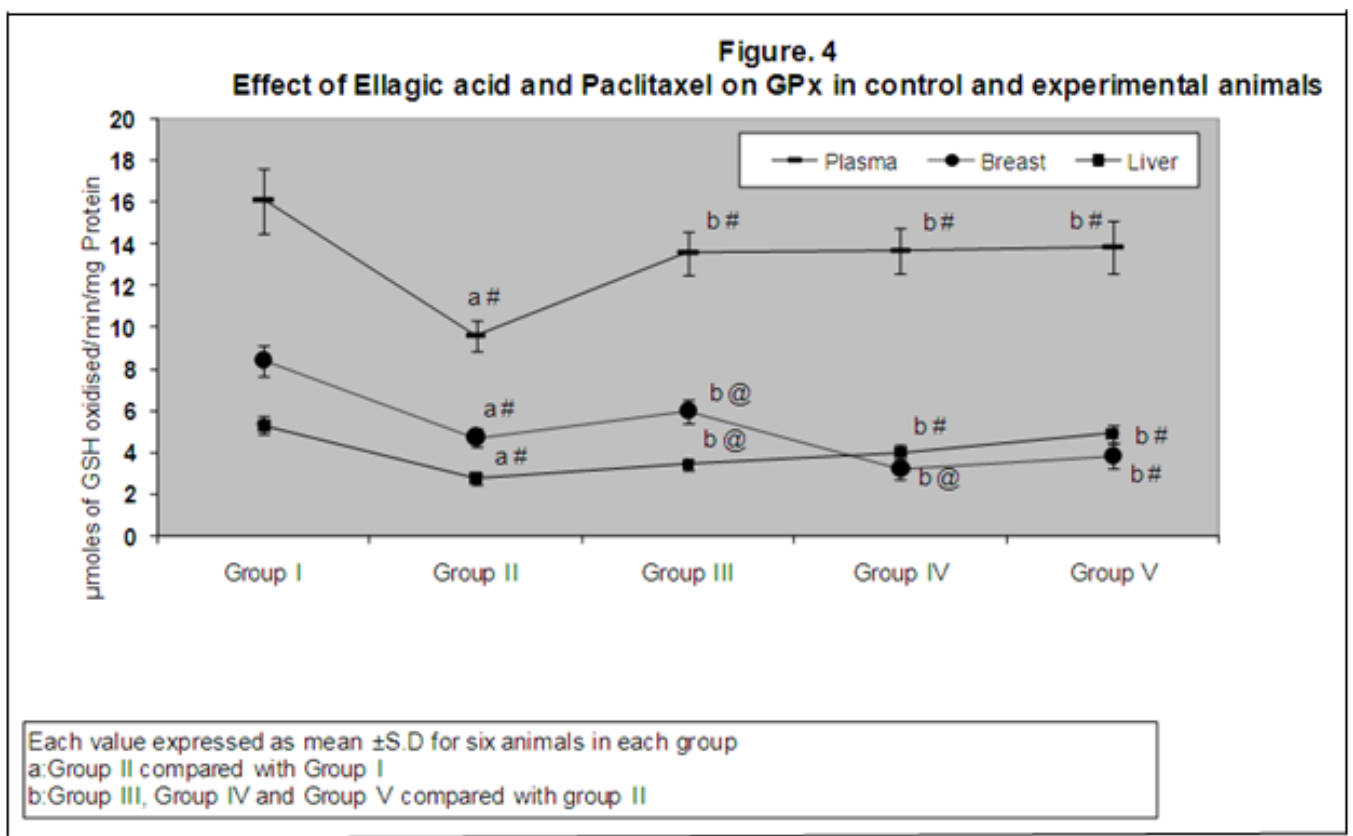

\section{References}

[1]. Agarwal O.P and Nagarathnam A.Radioprotective property of flavonoids in man. Toxicon.19,1981,201-204

[2]. Altieri DC, Survivin and molecular pathogenesis of colorectal cancer. Cancer Lancet, 362,2003, $205-209$.

[3]. Asai A , Expression level of Bcl-2 determines anti or proapoptotic function. Cancer Res, 59,1999, 4119-4128.

[4]. Bannasch P 1983Phenotypic instability in focal and nodular lesions induced in a short-term system in the rat liver. Carcinogenesis, 4,1983,595-603.

[5]. Birt DF Dietary agents in cancer prevention: Flavonoids and isoflavonoids. Pharmacol Ther,2001, $157-177$.

[6]. Bolden S 1998. Cancer statistic. CA Cancer J Clin, 48,1998m, 6-29.

[7]. Brillantes FP. Mammary cancer induced by a single feeding of polynuclear hydrocarbons and its suppression. Nature, 189,1961, 204-207.

[8]. Cholbi MR, Paya $\mathrm{M}$ and Alcaraz MJ.Inhibitory effect of phenolic compounds on CCl4 induced microsomal Lipid peroxidation.Experentia 47,1991,195-199.

[9]. Chu-cy,Chang JP,King Hong T,Wang CJ.Food Chem.Toxicol.32,1994,:373.1994

[10]. Claycomb WC 2005. Ultrastructure of terminally differentiated adult rat cardiac muscle cells in culture. Am J Anatomy, 164,2005, 113-131.

[11]. Clarks R , Breast cancer: New biological approaches to treatment. In: J Waxman (Ed.): Molecular Endocrinology of Cancer. USA: Cambridge University Press, 1996,271-304.

[12]. Daniel EM, The effect of ellagic acid and 13-cis-retinoic acid on N-nitroso benzylmehylamine-induced esophagal tumorigenesis in rats. Cancer Lett, 56,1991, 117-124.

[13]. Daniel P.S and Joyce NJ.DNA adduct formation by 7,12 dimethyl benz (a) anthracene and its non-carcinogenic 2-fluro analogue in female Sprague dawley rats.J.Natl.Cancer Inst.70,1983,111-118.

[14]. Desai ID.Vitamin E analysis methods for animal tissues. Meth.Enzymol.105,1984, 138-144.

[15]. Greenlee RT , Cancer statistics. CA Cancer J Clin, 51,2001,15-36. 
[16]. Gutteridge JMC,Halliwell B.The measurement and mechanism of lipid peroxidation in biological system.TIBS 15,1990,129-135.

[17]. Hazelton AG,Lang CA.Glutathione content of tissues in the aging mouse .Biochem J.188,1980,25-30.

[18]. Hu YF 2001. Cancer risk related to mammary gland structure and development. Microsc Res Tech, 52: 204-223.

[19]. Khanan A. 2005. Evaluation of toxicities induced by chemotherapy in breast cancer patients. Biomed Pharmacotherpy, 59: 524-527.

[20]. Kitagawa T 1986. Hepatocarcinogenesis in the rat: The effect of promoters and carcinogens in vivo and in vitro. Int. Rev. Cytol, 101: $125-173$.

[21]. Lowry OH, Rosebrough NJ, Farr AL and Randall RJ.Protein measurement with the folin-phenol reagent.J.Biol.Chem.193,1951, 265-275.

[22]. Marklund S and Marklund G.Involvement of superoxide anion radical in the autooxidation of pyrogallol and a convenient assay for Superoxide dismutase.Eur.J.Biochem.47,1974,469-474.

[23]. Matesich SM 2003. Second cancers after breast cancer treatment. Semin Oncol, 30: 740-748.

[24]. Morse MA 1993. Cancer chemoprevention: Principles and prospects. Carcinogenesis, 14: 1737-1746.

[25]. Moron MS, Depierre JW and Mannervik B.Levels of glutathione, glutathione reductase and glutathione -S-transferase activities in rat lung and liver.Biochem.Biophys.Acta.582,1979,67-68.

[26]. Ohkawa H,Ohnishi $\mathrm{N}$ and Yagi K.Assay for lipid peroxidation in animal tissues by thiobarbituric acid reaction .Anal.Biochem.95,1979,351-358.

[27]. Omaye ST, Turnbull JD and Sauberlich HE.Selected methods for the determination of ascorbic in animal cells tissues and fluids.Meth.Enzymol.62,1971,3-11.

[28]. Parkin DM, Pisani P, Ferlay J.Estimates of the worldwide incidence of 25 major cancers in ,Int.J.Cancer.80,1999,827-41.

[29]. Qiuo Z 2003. Flavonoids: Promising anticancer agents. Med Res, 23: 519-34.

[30]. Ratty A.K and Das NP.Effect of flavonoid on nonenzymatic Lipid peroxidation : Structure activity relationship.Biochemical Medicine and metabolic Biology.39,1998,69-79.

[31]. Rotruck JT,Pope AL,Ganther HE ,Swanson AB,Hafeman DG and Hoekstra WG.Selenium: biochemical role as a component of glutathione peroxidae purification and assay.Science.179,1973,588-590.

[32]. Rowinsky E.K.and Donehower R.C.Paclitaxel (Taxol). N.Eng.J.Med.332,1995,1004-1014

[33]. Russo J . Tumors of the Mammary Gland and pathology of tumors in laboratory animals. Lyon, 1996,47-48.

[34]. Russo IH 1978. DNA labeling index and structure of the rat mammary gland as determinants of its susceptibility to carcinogene sis. J Natl Cancer Inst, 61: 1451-1459.

[35]. Sinha AK.Colorimetric assay of Catalase.Anal.Biochem.47,1972,389-39

[36]. Sies H,Stahe W,Sundquist AR.Ann NY.Acad Sci.1992,669:70.

[37]. Sun Y. 1990.Free radicals antioxidants, enzymes and Carcinogenesis.Free Radical Biol Med.8,1990, 583-599.

[38]. Wiseman H.Dietary influences on membrane functions:importance $s$ in protection against oxidative damage and diseases.J.Nutri.Biochem.7,1996,2-15.

[39]. Winkler B.S.Uneqivocal evidence in support of the non enzymatic redox coupling between glutathione, glutathione disulfide ascorbic acid and dehydroascorbic acid.Biochem.Biophys Acta.1117 (3),1992, 287-290. 